\title{
ESTUDIO DE LA RESPUESTA INMUNE FRENTE A INFECCIONES GENITALES Y SU RELACIÓN CON IN- FERTILIDAD. CONTRIBUCIÓN AL DISEÑO DE ESTRATEGIAS INMUNOPROFILÁCTICAS CONTRA CHLAMYDIA TRACHOMATIS
}

Romina Cecilia Russi
rcrussi@fbcb.unl.edu.ar

Doctorado en Ciencias Biológicas

Directora: Dra. Carolina Veaute

Lugar de realización: Laboratorio de Inmunología Experimental, Facultad de Bioquímica y Ciencias Biológicas, Universidad Nacional del Litoral (FBCB-UNL)

Fecha de la defensa: 19 de marzo de 2019

\section{RESUMEN}

La infertilidad es un problema de salud pública que afecta a personas de todo el mundo. Una de las causas de infertilidad son las infecciones de transmisión sexual (ITS). La infección genital por Chlamydia trachomatis es una de las más prevalentes en todo el mundo y tiene un efecto significativo en la reproducción humana. Por otra parte, Mycoplasma hominis y Ureaplasma urealyticum son a menudo concomitantes con $C$. trachomatis y han sido descritos como microorganismos causantes de ITS que en diversas situaciones están estrechamente relacionados con trastornos de la salud reproductiva. El objetivo general fue investigar la relación entre la respuesta inmune asociada a infecciones genitales y la infertilidad, y contribuir al diseño de una estrategia de prevención de infección por $C$. trachomatis. En consecuencia, en primer lugar se analizó la prevalencia de infecciones genitales producidas por $C$. trachomatis, M. hominis y U. urealyticum en pacientes en consulta por infertilidad del hospital JB Iturraspe de la ciudad de Santa Fe. Luego se caracterizó la expresión de citoquinas (IFN- $\gamma$, TNF- $\alpha$, IL-17, IL-10, TGF- $\beta$, IL6, IL-8), y factores de transcripción maestros (FTM) de linfocitos T helper (LTh) (Th1: Tbet, Th2: GATA3, Treg: FOXp3 y Th17: RORyt) por RT-qPCR y se determinaron anticuerpos antiespermáticos (ASA) en lavado vaginal y en semen de pacientes infértiles y fértiles, con y sin estas infecciones genitales. Por otro lado, mediante citometría de flujo se cuantificaron IFN- $\gamma$, IL-10, IL-6, IL-17A. Con los resultados obtenidos se establecieron posibles relaciones entre el perfil de respuesta inmune hallado y la infertilidad. En segundo lugar, se diseñaron, optimizaron, desarrollaron y evaluaron diferentes formulaciones basadas en ADN plasmídico (ADNp) y proteína recombinante para inducir inmunidad hacia la proteína de membrana polimórfica $\mathrm{D}(\mathrm{PmpD})$ de $C$. trachomatis. Mediante un análisis in sílico se seleccionó un fragmento de PmpD denominado Fragmento 2 (F2), que contiene múltiples epitopes de linfocitos B, conformacionales y lineales, y de linfocitos T, con capacidad de unión a moléculas MHC II. La secuencia codificante del mismo fue insertada en un vector de expresión eucariota, precedida de un líder secretorio, para ser 
usado en la inmunización con ADNp. Asimismo, F2 se obtuvo por expresión recombinante en Escherichia coli. Con ambos inmunógenos se realizó un protocolo de inmunización prime-boost. Ratones hembra Balb/c recibieron una dosis con ADNp desnudo. Luego se administraron 2 refuerzos con F2 de PmpD recombinante por dos vías simultáneas (sistémica y de las mucosas), formulada con liposomas suplementados o no con un inmunoestimulante, Los inmunoestimulantes empleados fueron: oligodesoxinucleótidos (ODN) CpG y anfifilos Gemini (Gem). Para evaluar la respuesta inmune humoral generada se determinaron por ELISA los niveles de anticuerpos específicos IgG, IgG1 e IgG2a en suero e IgG e IgA en lavados vaginales. Por último, se evaluó la fertilidad in vivo de los animales inmunizados mediante el apareo con machos de fertilidad comprobada. Respecto al análisis de prevalencia, los resultados mostraron que el 29,9\% de los 193 pacientes infértiles analizados presenta alguna de estas tres ITS, indicando a las infecciones por $C$. trachomatis, $M$. hominis y $U$. urealyticum como potenciales causas de infertilidad. Particularmente, $C$. trachomatis y $M$. hominis son más prevalente en mujeres infértiles y $U$. urealyticum en hombres infértiles. Los resultados obtenidos sugieren que los ASA no serían los principales factores que contribuyen a la infertilidad en los pacientes estudiados. Sin embargo, tanto los pacientes infértiles con las ITS evaluadas como sin estas ITS presentaron alteraciones en citoquinas y FMT de LTh (particularmente se observó una predisposición a un ambiente proinflamatorio) tanto en lavado vaginal como en plasma seminal. Estos resultados apoyan la hipótesis de que las ITS producen alteraciones en el ambiente inmunológico que podrían impactar negativamente en la fertilidad. Por otro lado, las tres formulaciones vacunales evaluadas, generaron una robusta respuesta humoral, tanto sistémica como en mucosa vaginal en un modelo murino empleando la estrategia de prime-boost. Particularmente, la formulación que contenía F2 acompañada de liposomas catiónicos y ODN-CpG generó altos niveles de anticuerpos IgG2a séricos específicos lo que sugiere que este inmunoestimulante dirige la respuesta hacia un perfil Th1, e IgG en mucosa vaginal. Por otro lado, la formulación con liposomas y Gem condujo a la producción de anticuerpos de isotipo IgA en vagina. Los animales inmunizados no exhibieron alteraciones en los parámetros de fertilidad evaluados (potencial de fertilidad, tasa de pérdida pre- y pos-implantación). El presente trabajo de tesis permitió comprender los factores locales que regulan la inmunidad de las mucosas en pacientes infértiles con y sin infecciones genitales y diseñar, desarrollar y evaluar diversas formulaciones vacunales para lograr inmunidad contra $C$. trachomatis sin alterar la fertilidad. En conjunto, este trabajo brinda elementos para una mejor comprensión de los trastornos reproductivos y aporta posibles herramientas para la prevención de una ITS con gran impacto en la salud reproductiva y neonatal. 


\section{ABSTRACT}

Immune response against genital infections and its association with infertility. Contribution to the design of immunoprophylactic strategies against Chlamydia trachomatis

Infertility is a public health problem that affects people worldwide. Sexually Transmitted Infections (STI) are a common cause of human infertility. The general objective was to investigate the relationship between the immune response associated with Chlamydia trachomatis, Mycoplasma hominis and Ureaplasma urealyticum infections and infertility, and to contribute to the design of vaccines to prevent C. trachomatis infection. A prevalence analysis showed that $29.9 \%$ of the 193 infertile patients analyzed have any of these three STI. The study of the immune factors suggests that antisperm antibodies (ASA) would not be the main contributing factor to infertility in the patients studied. However, infertile patients (with and without STI) presented alterations the cytokines and master transcription factors expression in vaginal washing and seminal plasma. Taken together, these results support the hypothesis that STI produce alterations in the immune environment that could negatively impact fertility. Immunization assays using a prime-boost strategy against the Polymorphic membrane protein $\mathrm{D}(\mathrm{PmpD})$ from C. trachomatis, using different adjuvant formulations, generated a robust humoral response, both systemic and local (in vaginal mucosa) in a murine model, without negative effects upon fertility parameters. This work allowed understand the local factors that regulate the immunity in infertile patients and to design, develop, and evaluate various vaccine formulations to achieve protective immunity against C. trachomatis. Taken together, this work provides elements for a better understanding of reproductive disorders and provides possible tools for the prevention of STI with a great impact on reproductive and neonatal health. 